\title{
CARACTERIZACIÓN DEL DESARROLLO PSICOMOTOR DE NIÑOS Y NIÑAS CON CÁNCER
}

\author{
PSYCHOMOTOR DEVELOPMENT CHARACTERIZATION OF CHILDREN WITH
}

\section{Uribe-Echevarría M., L. ${ }^{1}$, Madrid V., B. ${ }^{2}$, Picand S., K ${ }^{3}$, Leiva J., A. ${ }^{4}$, Rojo S., L. ${ }^{5}$}

\section{RESUMEN}

Antecedentes: El desarrollo psicomotor (DPSM) es una condicionante social de la salud, por lo que sus alteraciones pueden perpetuar otras desigualdades hasta la adultez. Los menores con cáncer se ven expuestos a una serie de factores que pueden poner en riesgo su DPSM. Metodología: Se evalúa el DPSM de 35 niños y niñas con cáncer de 0 a 5 años de dos hospitales, con los test EEDP y TEPSI, estandarizados para población chilena. Se excluyen los niños/as con características o patologías de base que pudiesen explicar el retraso del desarrollo psicomotor (tumores cerebrales, discapacidad visual, síndrome de Down o hemiparesia.). Las evaluaciones se realizan en período ambulatorio estando los niños y niñas en buenas condiciones generales, sin infecciones activas y con parámetros hematológicos estables. Es decir, en igualdad de condiciones de salud, salvo por el diagnóstico oncológico de base. Resultados: El promedio nacional de rezago es 5,5\%, el de riesgo 5,64 y el retraso alcanza un 1,3\%. Sumando las tres categorías alcanza un 12,44\%. Los niños/as evaluados arrojan un 28,52\%, 14,29\% y 2,86\% respectivamente, con un total de 45,67\%, es decir 3,67 veces más alteraciones del DPSM que los niños sin cáncer. Conclusiones: Los niños y niñas con cáncer evaluados presentan más alteraciones del DPSM que aquellos que no tuvieron cáncer antes de los 5 años de edad. Existen iniciativas ya implementadas desde el 2007 por el Sistema de Protección Integral de la infancia para prevenir e intervenir las alteraciones del DPSM, pero parecen no se suficientes para esta población específica.

\section{Palabras Claves:}

cáncer infantil/pediátrico, desarrollo psicomotor, retraso

1 Terapeuta Ocupacional y Licenciada en Ciencias de la Ocupación Humana. Diplomada en Estrategias de Intervención Temprana en Salud Mental Infantil. Arteterapeuta. Unidad de Oncología Infantil, Complejo Asistencial Dr. Sótero del Río. Santiago, Chile. +56225764657 lo.uribe@gmail.com

2 Terapeuta Ocupacional y Licenciada en Ciencias de la Ocupación Humana. Unidad de Salud Mental Infanto-Juvenil. Hospital Militar. Santiago, Chile. beamadridv@gmail.com

3 Terapeuta Ocupacional y Licenciada en Ciencias de la Ocupación Humana. Cread Arrullo, Hogar de Lactantes y Pre escolares SENAME, Concepción, Chile. picand.katalina@gmail.com

$4 \quad$ Psicólogo. Unidad de Oncología Infantil, Complejo Asistencial Dr. Sótero del Río. Santiago, Chile. leiva.alvaro@gmail.com

5 Psicóloga. Master en Psicooncología. Unidad de Cuidados Paliativos Adultos, Complejo Asistencial Dr. Sótero del Río. Santiago, Chile. leca. rojo@gmail.com 


\begin{abstract}
Background: Psychomotor development (PD) is known to be a social determinant of health which means that a development delay or disorder can perpetuate other inequalities into adulthood. Children with cancer are exposed to a number of factors that may jeopardize their PD. Methodology: All eligible children with cancer under 5 years were evaluated in two hospitals of Santiago, with a total $n^{\circ}$ of 35. Children with underlying conditions that may explain a developmental delay were excluded (brain tumors, visually impaired, Down Syndrome). Evaluations were performed on outpatient period, in good general conditions, meaning in equal terms than children in the general population, except for the cancer. Results: The average national lag is 5.5\%, the risk 5.64 and the retardation reaches $1.3 \%$. Adding the three categories of delay, it reaches a $12.44 \%$. Children evaluated presented $28.52 \%, 14.29 \%$ and $2.86 \%$ respectively, with a total of $45.67 \%$, that means 3.67 times more developmental delays than children without cancer. Conclusions: Children with cancer have more PD disorders than those who did not have cancer before age 5. There are initiatives already implemented since 2007 by the System of Integral Protection of Children, with protocols to prevent and attend PD disorders, but seem to be insufficient for this specific population.
\end{abstract}

\title{
Keywords:
}

pediatric/child/childhood cancer, psychomotor development, delay

\section{INTRODUCCIÓN Y ANTECEDENTES}

El Ministerio de Salud de Chile (2008) afirma que "la desigualdad que se origina en la carencia de condiciones apropiadas para el desarrollo óptimo de niños y niñas, es una de las que marca la vida y perpetúa otras desigualdades como las asociadas a la pobreza y al género, indicando que los déficits en el desarrollo infantil temprano, en tanto determinante social de la salud, "tienen la capacidad de amenazar las competencias requeridas para vivir en sociedad".

El desarrollo psicomotor de los niños y niñas entre los 0 y los 5 años en Chile se previene, pesquisa y trata principalmente en la atención primaria. Los niños y niñas con cáncer no asisten a esos centros de atención por al menos un año y medio en su fase inicial de diagnóstico y tratamiento, por lo tanto, se desconoce su desarrollo psicomotor. En caso de mayores alteraciones como polineuropatías por vincristina u otros quimioterápicos o trastornos motores, cognitivos o de procesamiento en niños con tumores cerebrales, si podemos encontrar estadísticas y líneas de intervención, pero las posibles alteraciones del DPSM no se encuentran suficientemente evaluadas, escondidas tras las otras grandes dificultades agudas relacionadas con el cáncer y su tratamiento.

Los niños(as) con cáncer presentan múltiples factores que ponen en riesgo su desarrollo psicomotor (DPSM en adelante). Sin embargo, no es sólo el cáncer, sino también factores familiares, sociales, físicos o psicológicos, los que influyen fuertemente en las oportunidades de desarrollo de los menores. Teóricamente, podrían dividirse estos factores de riesgo en tres tipos: aquellos derivados del cáncer mismo (dolor óseo, epistaxis, anemia), aquellos derivados del tratamiento (inmovilización, aislamiento obligado, malestar general) y factores psicosociales (el miedo a sangrar, el ambiente desconocido, la falta de amigos, la sobreprotección).

Estudios realizados en sobrevivientes de cáncer infantil (Barahona et al, 2009, Martínez 2000) muestran que presentan importantes dificultades en su autocuidado en salud, en la integración social y en sus competencias de adaptación cotidiana.

Sabemos, entonces, que tanto los adultos que tuvieron cáncer en la infancia como los adultos que tuvieron retraso del desarrollo psicomotor (RDPSM en adelante) a temprana edad presentan dificultades en su inclusión y competencia psicosocial. Podríamos pensar que aquellos que tengan ambas condiciones, es decir, cáncer y RDSPM serán aún más perjudicados.

Siendo el RDPSM una alteración de salud "fácil y barata" de detectar e intervenir, mucho más que sus secuelas a largo plazo al menos (MINSAL, 2011), se hace fundamental la necesidad de objetivar esta condición y tratarla oportunamente. En palabras simples, probablemente no podemos evitar el cáncer, pero si podríamos evitar el retraso del desarrollo psicomotor en los niños con cáncer.

Tomando como ejemplo el Hospital Sótero del Río y utilizando el registro de los ingresos entre los años 2000 y 2010, encontramos que el 47,2 \% de los niños y niñas de la Unidad de Oncología Infantil corresponde a menores de 5 años. Esta cifra nos indica que cerca de la mitad de los niños con cáncer en nuestra población, se 
encuentra ante todos los factores de riesgo socioemocional y biológico que acarrea el cáncer en la edad de mayor trascendencia desde la perspectiva de desarrollo psicomotor, afectivo y cortical.

La pregunta de investigación que surge es la siguiente: ¿Cuál es el nivel de desarrollo psicomotor de los niños y niñas entre 0 y 5 años atendidos en una Unidad de Oncología Infantil? Esperamos poder categorizar ese nivel en normal, rezago, riesgo o retraso, y comprarlo con los niveles encontrados en la población nacional, para así conocer si existen diferencias entre el nivel de DPSM de niños(as) con y sin cáncer.

El objetivo general de este estudio es caracterizar el desarrollo psicomotor de niños y niñas que se encuentran en tratamiento por cáncer. Los objetivos específicos en relación a los niños y niñas entre 0 y 5 años evaluados que se encuentran en tratamiento por cáncer, se pretende: a) Categorizar su desarrollo psicomotor en normal, rezago, riesgo o retraso; b) Describir perfiles de presentación de déficit del desarrollo psicomotor; c) Caracterizar la percepción de los cuidadores sobre el desarrollo psicomotor de sus hijos/as.

Considerando que se tomarán niños y niñas hasta los 5 años, todos se encuentran dentro de los 5 años de garantías GES, y por lo tanto, para este estudio se considerará "que se encuentran en tratamiento por cáncer", estén o no con quimioterapia o radioterapia activa.

Para las categorías normal, rezago, riesgo o retraso se utilizaron las definiciones otorgadas por el Ministerio de Salud y el Sistema de Protección Integral de la Infancia a través del documento "Manual para el Apoyo y Seguimiento del Desarrollo Psicosocial de los niños y niñas de 0 a 6 años" (MINSAL 2008), basadas en las escalas otorgadas por los mismos tests.

Para este estudio, "déficit del desarrollo psicomotor" y "alteración del desarrollo psicomotor" será el nombre genérico utilizado para describir las tres categorías de desviación del puntaje normal, a saber, rezago, riesgo y retraso juntos. Esta consideración es importante, ya que en algunos textos se entiende por déficit únicamente las categorías de riesgo y retraso.

Este estudio fue realizado gracias al aporte de la Fundación Nuestros Hijos a través de sus fondos concursables de investigación. Esta fundación apoya de dos maneras: por un lado financia la compra del material necesario como baterías de los test, hojas de registro y consentimiento informado, y por otro lado, a través de un comité científico de profesionales del área de la Oncología y la Medicina Infantil Nacional, que revisa, apoya y guía a los investigadores en el diseño y ejecución del proyecto.

Considerando que la Fundación Nuestros Hijos es una institución sin fines de lucro, y que los aportes no consideran honorarios para los investigadores, no se presentan conflictos de intereses en relación a la fuente de financiamiento.

Respecto a los investigadores, no se consideran que existan conflictos de interés personales ni institucionales.

\section{MetodoloGía}

La investigación se dividió en dos grandes etapas. La primera contempló el acercamiento y la aplicación de los instrumentos en la población destinataria del Complejo Asistencial Dr. Sótero del Río. Este primer período se realizó entre los meses de julio a diciembre del año 2012. La segunda etapa, fue planificada para el primer semestre del año 2013, en los hospitales Dr. Luis Calvo Mackenna y Dr. Exequiel González Cortés (HEGC), pero sufrió modificaciones, y fue aplicada finalmente solo en el HEGC, por motivos que se describen más adelante.

Para aproximarse a conocer el nivel de desarrollo psicomotor y alcanzar el objetivo general de la investigación se realiza una investigación de tipo exploratorio - descriptivo que pretende dar a conocer características del desarrollo psicomotor de los niños y niñas con cáncer, más no causas, relaciones o asociaciones de las manifestaciones que se describan. Temporalmente se trata de una investigación transversal-seccional ya que se realizó un corte temporal, tomando a todos los niños menores de 5 años al momento de la evaluación, realizada en un lapso de tiempo contempla los meses entre julio 2012 y diciembre 2013.

La clasificación de niños/as en las categorías de desarrollo (normal, rezago, riego y retraso), así como el análisis de la percepción de los cuidadores respecto al desarrollo psicomotor de sus hijos serán analizados en términos de frecuencia y porcentajes, por lo tanto de manera cuantitativa.

El universo de este estudio contempla a todos los niños y niñas menores de 5 años atendidos por cáncer en las unidades Oncología Infantil de tres de los seis centros del Programa Nacional Infantil de Drogas Antineoplásicas (PINDA) de la Región Metropolitana; Complejo Asistencial Dr.Sótero del Río (CASR), Dr. Exequiel 
González Cortés (HEGC) y Dr. Luis Calvo Mackenna (HLCM).

Actualmente existen 13 centros PINDA distribuidos a lo largo del país, que atienden a todos los beneficiarios del sistema público de salud, que ascienden a aprox. 420 de los 510-590 casos nuevos anuales (Campbell, 2005).

Se escogen los Hospitales Dr. Sótero del Río, Dr. Calvo Mackenna, Dr. Exequiel González Cortés por razones de conveniencia, ya que por la cercanía con miembros del equipo de oncología infantil de dichos establecimientos se facilita la coordinación y aplicación de la investigación.

Los Hospitales CASR y HEGC presentan características similares en cuanto a tipo de población, recursos humanos e ingresos anuales, y el hospital HLCM incluye también población de otras regiones de Chile, lo que se considera interesante de observar.

En relación a los aspectos éticos es importante mencionar que el estudio fue presentado y aprobado por los comités de ética de los servicios de salud correspondientes a los tres hospitales. Las evaluaciones fueron iniciadas una vez realizadas las modificaciones solicitadas a los consentimientos informados y recibidas las actas de aprobación.

Dado que la muestra son menores de 5 años que aún no manejan habilidades de lecto-escritura, no se aplicó asentimiento informado a los niños/as participantes, pero si se explicó verbalmente la evaluación a realizar.

Se realizó un consentimiento informado al cuidador principal del menor a evaluar, describiendo tanto la aplicación del test del desarrollo psicomotor como la participación en la investigación. Esta autorización fue discutida con el cuidador principal en presencia de un testigo.

En los documentos de consentimiento también se incluye la aceptación o el rechazo de la utilización de fotografías del menor para poster o presentaciones. La elección o declinación de este permiso es independiente del de la aplicación del test.

Además, debido a que la evaluación podría arrojar resultados importantes para el futuro del menor evaluado, al cuidador principal se le entregó una copia del test, de la hoja de resultados y un recibo de los mismos que además incluye las indicaciones a seguir.

Debido a errores de metodología de aplicación de los test, registro y cálculo de datos, se suspende la investigación en el HLCM y se utilizan solamente los datos de los hospitales CASR Y HEGC. Dicho proceso fue supervisado por el comité de ética correspondiente y se realizó un protocolo de reparación con las familias del HLCM que habían sido evaluadas, que también fue aprobado por dicho comité.

Finalmente se incluyeron en la muestra a todos los niños y niñas que al momento de la evaluación tenían entre 0 días y 4 años 11 meses y 29 días. El $n^{\circ}$ total alcanzado fue de 35 niños. Se excluyeron todos los niños y niñas que presentaban una edad cronológica igual o superior a 5 años 0 meses 0 días. Se excluyeron también a niños y niñas que presentaban otras condiciones que podrían explicar el retraso del desarrollo psicomotor como trastornos neuromotores (hemiparesia, sd. cerebeloso, parálisis cerebral), gliomas del nervio óptico, retinoblastomas, cuidados paliativos en etapa avanzada, entre otros.

Se utilizaron cuatro instrumentos de evaluación; dos de ellos corresponden a Escalas de Evaluación del Desarrollo Psicomotor estandarizadas y dos a evaluaciones complementarias creadas para esta investigación en particular.

Las escalas de evaluación del desarrollo psicomotor escogidas fueron TEPSI y EEDP, evaluaciones que se encuentran validadas para la población chilena y que son las que actualmente están definidas como parte de las prestaciones universales que se realizan a los niños y niñas de en la atención primaria en el marco del Sistema de Protección Integral de la Infancia (MINSAL, 2008).

La Escala TEPSI (Test de Desarrollo Psicomotor 2 5 años) desarrollado por Haeussler y Marchant (2011) es un test estandarizado que evalúa el desarrollo psicomotor de niños entre 2 y 5 años de edad. Evalúa al niño en tres aéreas: coordinación, lenguaje y motricidad, mediante la observación de la conducta del niño frente a situaciones propuestas por el examinador. El tiempo de administración del instrumento puede variar según la edad y colaboración del niño entre 30 y 40 minutos.

La Escala de Evaluación del Desarrollo Psicomotor (EEDP) (Rodríguez y Cols, 2012) se utiliza en niños de 0 a 2 años de edad. Se observan las conductas del niño frente a situaciones específicas presentadas por el examinado, además de preguntas dirigidas a la madre o al acompañante del niño sobre este ante situaciones concretas.

Todos los niños y niñas fueron evaluados según las condiciones requeridas por el manual del MINSAL 
(2008), es decir, en buen estado general, sin fiebre, sin hambre, sin sueño, sin cuadro agudo. La única diferencia con la población general fue la presencia de la patología oncológica de base. Algunos niños/as eran portadores de catéter venoso central al momento de la evaluación, pero ninguno poseía otro tipo de vía venosa que pudiese alterar su desempeño.
En la TABLA 01 se muestran las equivalencias de cada los puntajes de ambos test a las diferentes categorías.

\section{TABLA 01: EQUIVALENTES EEDP Y TEPSI PARA CATEGORÍAS DE DPSM}

\begin{tabular}{|c|c|c|}
\hline Categoría & EEDP (en Puntaje Estándar) & TEPSI (en puntaje T) \\
\hline RETRASO & Menor o igual a 0.69 & Menor a 20 \\
\hline RIESGO & Entre 0.84 y 0.70 & Entre 20 y 30 \\
\hline NORMAL & Mayor o igual a 0,85 & Mayor a 30 \\
\hline REZAGO & $\begin{array}{c}\text { Puntaje global normal, pero con al menos 1 } \\
\text { área bajo el mes base cronológico }\end{array}$ & $\begin{array}{c}\text { Puntaje global normal, pero con al menos 1 área } \\
\text { con puntaje T menor a 30 }\end{array}$ \\
\hline
\end{tabular}

Los dos instrumentos complementarios confeccionados con propósito de este estudio fueron una ficha de individualización y una pauta de percepción del cuidador.

La ficha de individualización consistió en una ficha de registro de datos personales y antecedentes relevantes del tratamiento y la familia, como fecha de diagnóstico, tiempo transcurrido desde el diagnóstico, etapa de tratamiento actual, edad actual, edad al diagnóstico, entre otros.

La pauta de percepción del cuidador consistió en una escala tipo Likert (Fernández, 2009) para conocer sus actitudes hacia el cuidado y estimulación del desarrollo de los menores.

Se crea este instrumento para realizar un acercamiento inicial hacia las actitudes del cuidador que puedan influir en el desarrollo psicomotor del menor.

Los ítem fueron construidos en base a siete dimensiones, que se sugiere (Losada, 2006) son aspectos relacionales, de actitud o contextuales que tienen influencia significativa en la relación cuidador - persona cuidada. Las dimensiones son:

- $\quad$ Sobreprotección

- percepción del niño/a como frágil o vulnerable

- $\quad$ percepción de habilidades/dificultades del menor

- estrés percibido ante la situación de cuidarlo
- $\quad$ sensación de ineficacia ante el cuidado

- percepción del ambiente como apoyador, abandonador u hostil.

Se presentan 37 ítem, cada uno como una frase a la que el encuestado debe responder según una de las cuatro opciones de respuesta; Totalmente en desacuerdo, en desacuerdo, de acuerdo, totalmente de acuerdo. No se otorga la posibilidad de respuesta neutra (indiferente), ya que se busca poder observar una tendencia.

La escala fue pre-testeada en 10 cuidadores de niñas/os que no formaban parte del estudio, pero si de una población similar, es decir a cuidadores de niños/as mayores de 5 años de la Unidad de Oncología Infantil, con el objetivo de verificar la claridad de las afirmaciones.

Para la aplicación de los instrumentos de evaluación se contemplaron cinco evaluadores. Dos de ellos fueron las co- investigadoras y tres de ellas fueron Terapeutas Ocupacionales contratadas como personal de apoyo.

La validez inter evaluador tanto del EEDP como del TEPSI esta descrita dentro del proceso de validación explicado en sus manuales como alta (Haeussler y Marchant, 2011, Rodríguez \& Cols, 2012). De todas formas de realizó una capacitación a los cinco evaluadores para establecer criterios comunes específicos, protocolizar la entrega de información acerca del proyecto, especificar aspectos éticos y administrativos y revisar 
la metodología de aplicación, registro y cálculo de las escalas, y la recogida de datos de la ficha de individualización y escala de Likert.

\section{Resultados}

Consecuentemente con lo descrito en la literatura (Campbell, 2005, Vargas, 2000, Siegel, 2013), se presenta en la muestra un número significativamente mayor de leucemias.

Como es posible observar en la TABLA 02 y en los GRÁFICOS 01 y 02, que comparan datos epidemiológicos del Ministerio de Salud de Chile (MINSAL, 2012, pág. 165) con los datos obtenidos en las evaluaciones realizadas en este estudio, tanto los valores por cada categoría de déficit como el total de alteraciones del DPSM evaluadas es mayor en los niños con cáncer a los que se le aplicó la escala que en la población general.

El promedio nacional de rezago es 5,5\%, el de riesgo 5,64 y el retraso alcanza un 1,3\% (MINSAL, 2012, pág. 165). Los niños(as) oncológicos evaluados arrojan un $28,52 \%, 14,29 \%$ y $2,86 \%$ respectivamente. El total de déficit del DPSM para la población normal, sumando los porcentajes de rezago, riesgo y retraso es de 12,44\%. (TABLA 02). Los niños(as) con cáncer de este estudio presentan un promedio que asciende hasta el $45,67 \%$.

TABLA 02: COMPARACIÓN REZAGO, RIESGO Y RETRASO EVALUADO EN MENORES ONCOLÓGICOS V/S PROMEDIO NACIONAL (MINSAL, 2012, pág. 165).

\begin{tabular}{|c|c|c|c|c|}
\hline & rezago & riesgo & retraso & TOTAL DÉFICIT \\
\hline promedio nacional & 5,5 & 5,64 & 1,3 & 12,44 \\
\hline promedio oncología & 28,52 & 14,29 & 2,86 & 45,67 \\
\hline
\end{tabular}

GRÁFICO 01: PORCENTAJE DE REZAGO, RIESGO Y RETRASO DEL DESARROLLO PSICOMOTOR EVALUADO EN MENORES ONCOLÓGICOS V/S PROMEDIO NACIONAL (MINSAL, 2012, pág. 165).

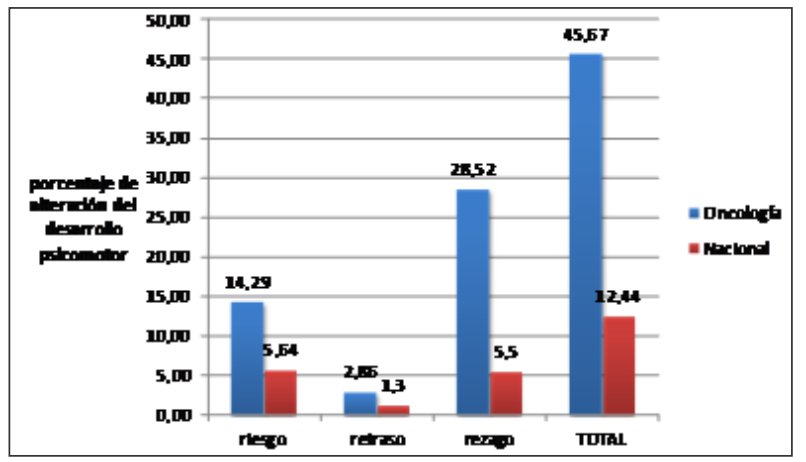

GRÁFICO 02: PORCENTAJE DE REZAGO, RIESGO Y RETRASO DEL DESARROLLO PSICOMOTOR EVALUADO EN MENORES ONCOLÓGICOS V/S PROMEDIO NACIONAL (MINSAL, 2012, pág. 165).

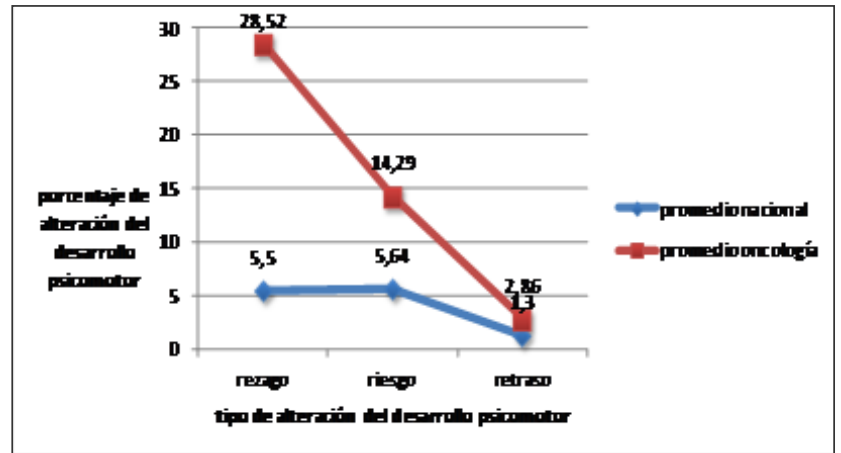


Al comparar los porcentajes de alteraciones del DPSM encontradas en la población normal con la muestra de niños con patología oncológica, encontramos que los niños y niñas con cáncer, presentan 5,19 veces más rezago, 2,53 veces más riesgo y 2,2 veces más retraso y del desarrollo psicomotor que los niños sin cáncer.

El resumen de los resultados globales de los test aplicados arroja que los niños y niñas con cáncer de la muestra presentan en total $1 / 3$ más $(33,23 \%)$ de déficit del desarrollo psicomotor que los niños y niñas sin cáncer, es decir 3,67 (casi 4) veces más.

Las alteraciones del desarrollo psicomotor se concentran especialmente entre los 13 a 18 meses desde el diagnóstico. Más del $80 \%$ de los menores evaluados en ese período presentaron alguna alteración del desarroIlo psicomotor. Significativos también son los períodos entre los 19 y los 30 meses, momento en el cual la mitad de los niños evaluados presenta alguna alteración.

En el GRÁFICO 03 podemos observar que gran parte de estas alteraciones corresponden a rezago. A medida que transcurre el tiempo desde el diagnóstico el rezago disminuye, el retraso se mantiene estable pero el riesgo de alteraciones del desarrollo psicomotor aumenta.

GRÁFICO 03: PORCENTAJE DE TIPO DE DÉFICIT DEL DESARROLLO PSICOMOTOR V/S MESES DESDE EL DIAGNÓSTICO

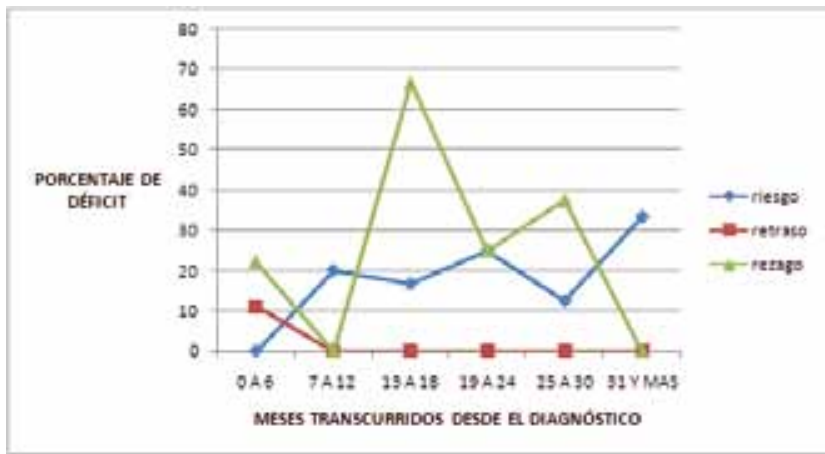

GRÁFICO 04: PORCENTAJE DE ALTERACIONES DEL DESARROLLO PSICOMOTOR SEGÚN TIPO DE ALTERACIÓN Y ETAPA DE TRATAMIENTO

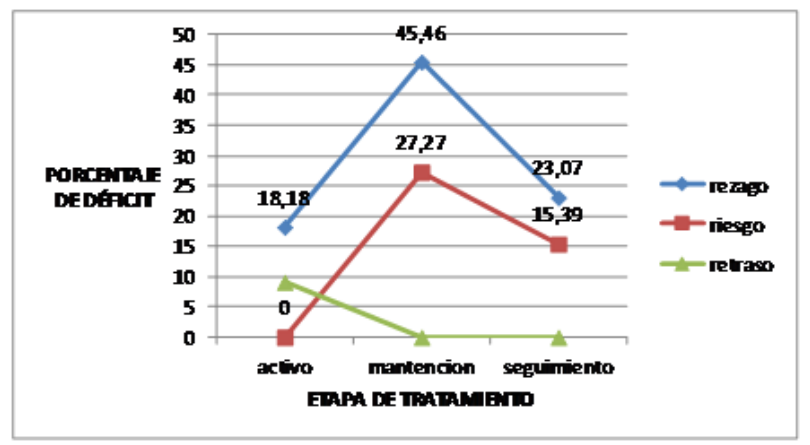

El rezago y el riesgo se observa que en la muestra aumentan durante la mantención, mientras que el retraso se mantiene o disminuye a medida que avanza el tratamiento (GRÁFICOS 03 y 04).

Al analizar la curva de déficit total del DPSM según edad en meses al momento del diagnóstico oncológico, podemos observar que los menores diagnosticados entre los 13 y 36 meses presentan mayores alteraciones del DPSM que los diagnosticados antes de los 12 o después de los 36 meses.

De gran sorpresa resulta el valor arrojado al especificar las áreas que se encuentran alteradas en los niños y niñas con rezago; la mitad corresponde al área motora y la otra mitad al área del lenguaje. No se pesquisan rezagos en el área de la coordinación (GRÁFICO 05).

\section{GRÁFICO 05: PORCENTAJE DE REZAGO SEGÚN ÁREA ALTERADA}

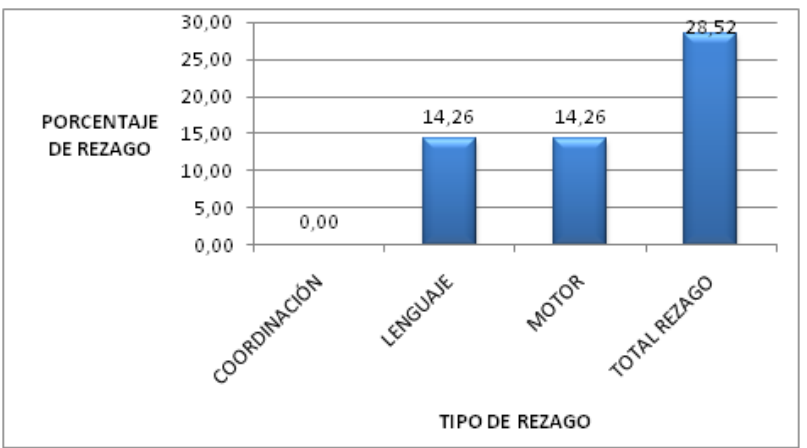


En relación al instrumento complementario aplicado, la pauta de percepción del cuidador, los resultados obtenidos fueron poco concluyentes.

Fueron eliminadas todas aquellas preguntas con respuestas dudosas, con más de dos alternativas marcadas o que fueron marcadas como poco comprensibles en el pre test. Además, se eliminaron 4 hojas de respuestas completas por encontrarse ilegibles, por lo que el $n^{\circ}$ de esta parte del análisis corresponde a 31 casos.

Como fue mencionado en la descripción de los instrumentos, la pauta de percepción del cuidador fue construida en base a dimensiones que se postula (Losada, 2006) que pueden influir en la calidad de la interacción entre usuario y cuidador.

El análisis de las respuestas de realizó por dimensión, agrupando los 37 ítem en las 6 dimensiones descritas anteriormente. Cada grupo de preguntas se refleja en una afirmación tipo. Por ejemplo, las frases "es muy difícil cuidar a mi hijo", o "me cuesta mucho duchar a mi hijo" son agrupadas en la dimensión 5: sensación de eficacia ante el cuidado, que es representada con la frase "me percibo a mí mismo como poco eficaz en los cuidados de mi hijo". De esta manera, se obtuvieron tendencias de la percepción de los cuidadores en relación a estas 6 dimensiones.

En términos generales, los cuidadores presentan percepciones acerca de la relación de cuidado que pueden interpretarse como positivas, ya que como perciben a los niños/as y a sí mismos como hábiles (GRÁFICOS 8 y 10) y al ambiente como apoyador (GRÁFICO 11). Tampoco se observa un perfil que describa el cuidado de los niños/as con cáncer como marcadamente estresante (GRÁFICO 9). La única dimensión que no presenta una tendencia clara es la de sobreprotección, con porcentajes de respuesta similares en todas las opciones de acuerdo o desacuerdo (GRÁfICO 06).

\begin{tabular}{|l|c|}
\hline $\begin{array}{c}\text { GRÁFICO 06: DIMENSIÓN 1: } \\
\text { SOBREPROTECCIÓN }\end{array}$ & $\begin{array}{c}\text { GRÁFICO07: DIMENS IÓN 2: PER CEPCIÓN DEL } \\
\text { NIÑO COMO FRÁGIL O VULNERABLE }\end{array}$ \\
\hline
\end{tabular}

\begin{tabular}{|c|c|c|c|c|c|c|c|c|c|}
\hline \multicolumn{5}{|c|}{$\begin{array}{c}\text { "Sobreprotejo más a mi hijo/a desde } \\
\text { que se enfermó" }\end{array}$} & \multicolumn{5}{|c|}{$\begin{array}{c}\text { "Percibo a mi hijo/a como frágil o } \\
\text { vulnerable" }\end{array}$} \\
\hline$x$ & & & $2 b . ;$ & 28,3 & $\therefore$ & Is & & & \\
\hline$m$ & $2:$. & $2 \xi, 3$ & & & 4:: & & & & \\
\hline 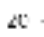 & & & & & का. - & & $2: 4$ & & \\
\hline 1: & & & & & $\because:-$ & & & i1 & \\
\hline 16 & & & & & $15-$ & & & & 3 \\
\hline 5 & & & & & $\begin{array}{c}16 \\
5\end{array}$ & & & & \\
\hline & 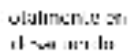 & er vesutucrso & De Aturte & 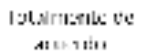 & & 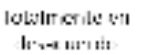 & 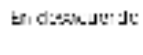 & W:A:verde & 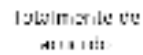 \\
\hline
\end{tabular}




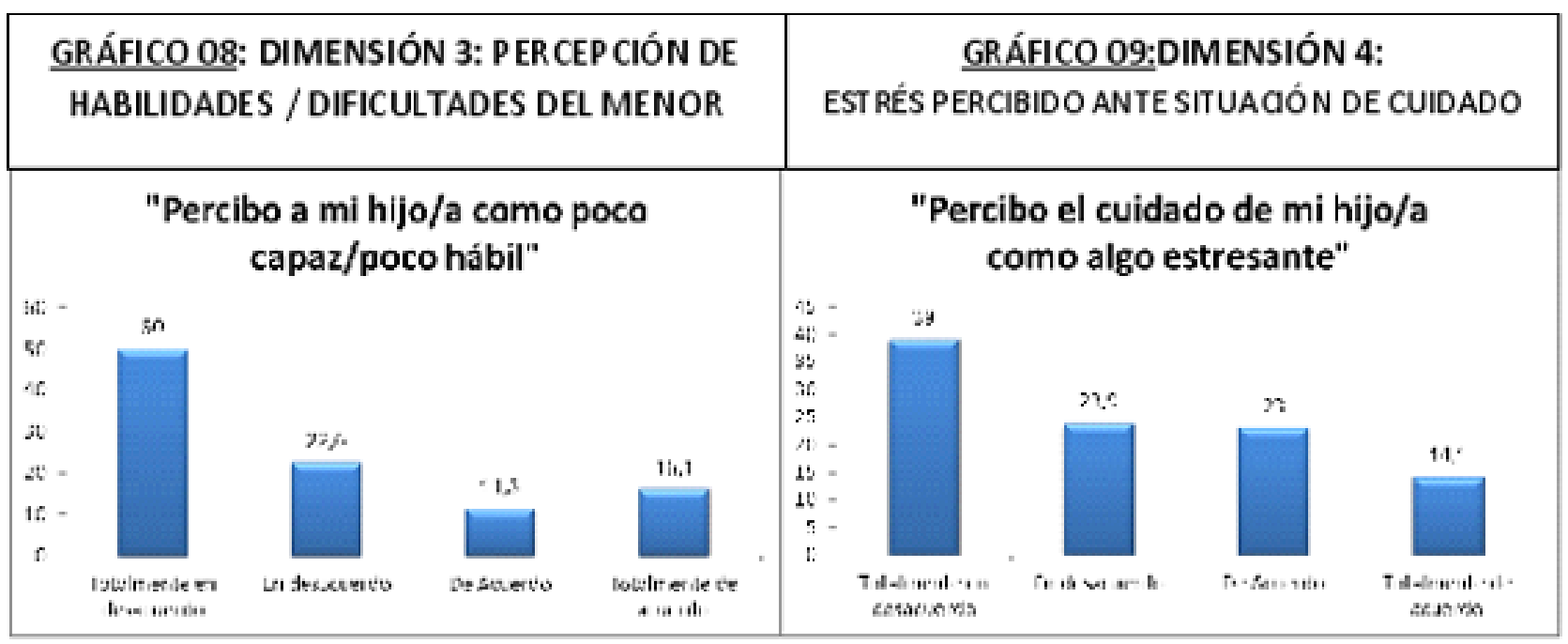

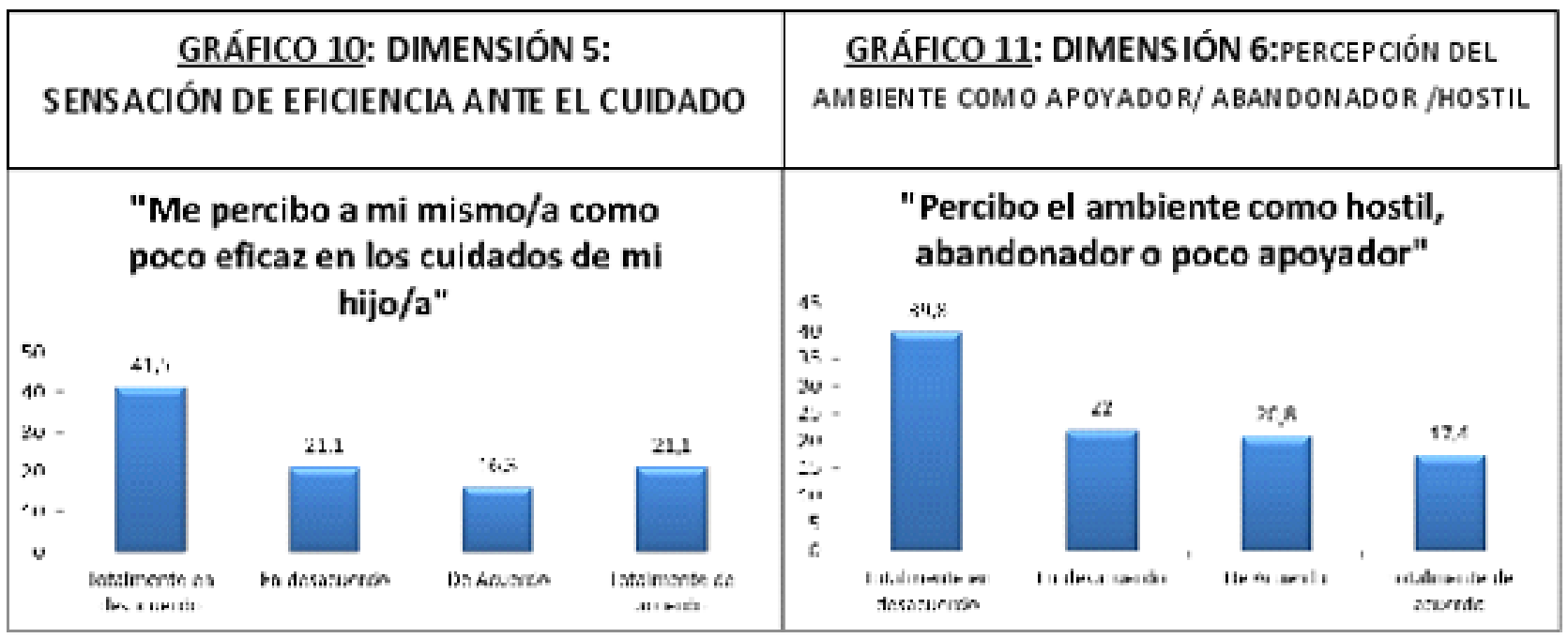




\section{DisCUSIÓN}

En Chile cerca del $70 \%$ de los niños tratados de cáncer sobrevive (Campbell, 2005). Esto se traduce en aproximadamente 390 sobrevivientes al año. Si pudiéramos generalizar los resultados de esta investigación, implicaría que un $45.7 \%$ de ellos, es decir, 178 de ellos podrían presentar alteraciones en el desarrollo, lo que podría llevar a perpetuar otras desigualdades a largo plazo.

La junta de expertos reunida en Erice, Sicilia el 2007 declaró que "La meta a largo plazo respecto a la cura y cuidado del niño con cáncer es que él / ella lleguen a ser adultos resilientes, activos y autónomos con una calidad de vida optima respecto a la salud y que sean aceptados por la sociedad al mismo nivel que sus pares" (Haupt, R. et al., 2007).

En la misma perspectiva, el documento Orientaciones Técnicas para las modalidades de apoyo al desarroIlo infantil el MINSAL $(2012$, p.11) postula que

"la vigilancia del desarrollo en los tres primeros años de vida será esencial porque cualquier alteración en esta etapa tendrá un impacto negativo en el logro del máximo potencial del desarrollo infantil. Además, el retorno económico de intervenciones en estos años, es muchísimo mayor que intervenciones que se realizan en etapas posteriores.

De este modo, el Ministerio de Salud y el Ministerio de Desarrollo Social a través de Chile Crece Contigo, se preocupa activamente no sólo de que los niños(as) chilenos estén sanos, sino que además puedan alcanzar su máximo potencial de desarrollo integral."

Podemos observar que tanto los expertos internacionales como el Gobierno de Chile concuerdan en que ya no solo es importante que los niños se curen del cáncer, sino que logren un desarrollo integral. La interrogante es de qué manera esto se está llevando a cabo, o de qué manera podría mejorarse su implementación.

Actualmente, existen 13 centros de tratamiento del cáncer infantil a nivel de Salud Pública en Chile. Esta red PINDA se encuentra formada desde el año 1988. Todos los centros cuentan con pediatras hemato-oncólogos. La mayoría cuenta con enfermeras especializadas en oncología. Sólo dos centros destinan horas especiales al seguimiento después del tratamiento. Sólo dos centros cuentan con trabajadora social de dedicación exclusiva. No todos los centros cuentan con psicólogo para la atención de los niños y sus familias. Dos centros cuentan con Terapeuta Ocupacional, uno por 44 horas y otro por 11 horas. Podría traducirse desde esta dotación que si bien se incluyen algunos profesionales que apoyan el desarrollo integral del niño y niña con cáncer, el énfasis sigue puesto en la curación y tratamiento médico.

A futuro, estudios que permitan observar el DPSM y sus principales factores de riesgo en los niños(as) con cáncer, aportarían significativamente al desarrollo de estrategias para prevenirlo, así como investigaciones relacionadas con el estrés crónico y sus influencias en el aprendizaje en el contexto del cáncer infantil.

Por otro lado, sería interesante volver a medir el desarrollo psicomotor en períodos posteriores a 5 años de tratamiento, para conocer la evolución del déficit pesquisado.

Tanto del proceso de investigación como de sus resultados surgieron nuevas reflexiones e interrogantes. Los niños y niñas con cáncer evaluados no solamente presentan mayor déficit que la población general, sino también se encuentran en un contexto y momento difícil de intervenir, que requiere comprensión del proceso de enfermedad del cáncer, de la situación, aprehensiones y funcionamiento familiar, sensibilidad especial y sobretodo flexibilidad.

Participar directamente de las evaluaciones en el CASR, así como implementar el protocolo de reparación en el HLCM permitieron a las investigadoras vivenciar lo difícil que puede ser sólo evaluar el DPSM de los menores con cáncer, no por la aplicación de las pruebas, sino por un sinfín de consideraciones prácticas y administrativas: A modo de ejemplo se describen algunas: Citarlos un día especial sólo para la realización del test no era factible, ya que la mayoría ya iba tantos días al hospital que no quería asistir voluntariamente a otra evaluación. Por lo tanto, eran citados el mismo día que ya tuvieran algún otro control médico, fuese en oncología o en otra especialidad. Y fueron innumerables las veces que hubo que posponer o modificar la hora de la evaluación por diversas razones; porque había que repetir un examen, porque aún no los atendía el médico, porque cambiaron el día del control, o porque el niño llegó con fiebre, o simplemente porque no se presentó, etc. La vida del niño/a con cáncer es difícil de planificar, ya que las condiciones pueden variar muy rápidamente, y el tratamiento no deja mucho espacio para otras actividades.

Se podría pensar entonces en educar a los padres, ya que al estar más tiempo con ellos estarán también presentes en los mejores momentos de alerta y ánimo para estimular a los menores. Sin embargo, los padres no siempre están en condiciones emocionales de re- 
cibir aún más información e instrucciones. Algunos lo logran, pero otros necesitan mayor acompañamiento y modelado (Puchol, 2011), es decir la presencia de otra persona que les muestre como relacionarse con sus hijos enfermos desde la parte sana, en ese nuevo contexto.

La atención del desarrollo psicomotor de los niños y niñas con cáncer no puede ser llevada a cabo por cualquier profesional, ya que requiere conocimientos y formación específicos en el área dada la complejidad del cuadro, tanto en relación a la patología misma, sus cuidados y tratamientos, como en relación al contexto social y familiar del niño con cáncer, ya que la familia se encuentra pasando por una crisis no normativa, y requerirá apoyos especiales para adaptarse a la nueva situación de salud y de vida.

Tal vez por eso en hospitales como el St Jude's Children Research Hospital en Estados Unidos cuentan con profesionales cuya misión es hacer de puente, traductor y herramienta entre el niño y el mundo del cáncer. Los "Child Life Specialist" (Thompson 2009, Council 2006, Burns-Nader, S., \& Hernandez-Reif, 2014) son profesionales del área infantil provenientes de la salud y la educación, que se forman luego dirigidamente en desarrollo infantil. En Chile no contamos con este tipo de formación, pero contamos con los terapeutas ocupacionales, que conocen y comprenden el mundo biomédico, y además poseen conocimientos profundos sobre el desarrollo infantil y la forma en que los niños y niñas crecen a través de las acciones que emprenden y de su relación con el entorno, y son por lo tanto, profesionales idóneos para esta labor.

Según lo observado y acorde a los resultados obtenidos, se pueden describir algunos factores importantes a considerar al momento de planificar una intervención para favorecer el desarrollo psicomotor de los niños y niñas con cáncer; el lugar y el tiempo de intervención deben ser flexibles, deben ser realizadas por un profesional especializado, deben considerar la crisis no normativa familiar y las implicancias relacionales y familiares que tiene en la estimulación del menor, debe iniciarse antes de los 13 meses desde el diagnóstico (luego aumentan las alteraciones) y debe dar énfasis en la estimulación de las áreas motora y del lenguaje.

En relación a las limitaciones de este estudio debe mencionarse sin lugar a dudas, que si bien la muestra está constituida por todos los niños evaluables de 2 de los 13 centros PINDA del país, el $\mathrm{n}^{\circ}$ de 35 evaluados es pequeño y no permite generalizar los resultados obtenidos.
Este estudio abre una línea de investigación en la que se pretende plantear, a largo plazo, que es posible favorecer y mejorar el desarrollo psicomotor de niños y niñas con cáncer de manera independiente a su condición oncológica. Sabiendo, a través de las materias dispuestas por la OMS (Macinko, Montenegro, Nebot, \& Etienne, 2007) que el desarrollo infantil temprano continúa influyendo en las personas en todo su ciclo vital, la intervención temprana en este medio no solo pondría atajo en esta alteración, sino que podría devolverle al niño o niña en tratamiento o ya tratado por cáncer, una herramienta fundamental para enfrentarse a las competencias de la vida en sociedad.

\section{ReFERENCIAS BibLIOGRÁficAS}

Aksarina, M. (1983) L'educació dels infants fins als set anys. Rosa Sensat, Barcelona. 37/261

Barahona, T., Cañete, A., Montero, M.L., Castel, V. (2009) Consulta de Seguimiento Activo de supervivientes de cáncer pediátrico. Revista Psicooncología Vol 6, núm 2-3, pp 373-379.

Barrera, F., Moraga, F., Escobar, S., Antilef, R.(2007) Participación de la madre y la familia en la atención del niño hospitalizado: Análisis histórico y visión de futuro. Revista Chilena de Pediatría, volumen 78, número 1.

Bernabeu, J. (2009) Atención Interdisciplinar a las secuelas de la enfermedad y/o tratamientos en oncología pediátrica. Psicooncología, Vol.6, Núm. 2-3, pp 381 -411.

Burns-Nader, S., \& Hernandez-Reif, M. (2014). Facilitating Play for Hospitalized Children Through Child Life Services. Children's Health Care, (just-accepted).

Campbell, M. (2005). Desarrollo de la Oncología pediátrica en Chile. Pediatría (Santiago de Chile), 2(2).

Council, C. L. (2006). Child life services. Pediatrics, 118(4), 1757-1763.

Diaz, N. (2001) Influencia de la Terapia Ocupacional y Psicomotricidad en Oncología Pediátrica. Universidad de Castilla - La Mancha. Facultad de Terapia Ocupacional, Logopedia y Enfermería.

Elejabarrieta, F., Iñiguez, L. (1984) Construcción de Escalas de Medición Tipo Thurst y Likert. U.A.B.

Elman, J., et al. (1998) Rethinking Innateness: A Connectionist Perspectiv on development. MIT Press

Erikson, E. (1950). Childhood and society. New York: Norton.

Erikson, E. (1985). The life cycle completed. New York: Norton.

Fernández de Pinedo, I. (2009) Construcción de una Escala de Actitudes Tipo Likert. Ministerio de Trabajo y Asuntos Sociales, España.

Haeussler, I., Marchant, T.(1985) TEPSI. Test de Desarrollo Psicomotor de 2 - 5 años. Ediciones Universidad Católica. 
Haeussler, I., Marchant, T.(Ed) ( 2011) TEPSI Test de Desarrollo Psicomotor 2-5 años. Santiago, Chile: Ediciones Univseridad Católica.

Haupt, R. et al. (2007) Declaración de Erice -Sobrevivientes a largo plazo de cáncer Infantil: Cura y Cuidado. European Journal of Cancer. Volume 43,Issue 12, págs. 1778-1780

Losada, A., Montorio, I., Izal, M., Márquez, M. (2006) Estudio e intervención sobre el malestar psicológico de los cuidadores de personas con demencia. El papel de los pensamientos disfuncionales. Madrid, España: Ministerio de Trabajo y Asuntos Sociales.

Macinko, J., Montenegro, H., Nebot, C., \& Etienne, C. (2007). La renovación de la atención primaria de salud en las Américas. Rev Panam Salud Publica,21(2-3), 73-84.

Martinez, A.M. (2000) Complicaciones a corto y largo plazo de los tratamientos oncológicos. Unidad de Oncología Pediátrica, Hospital Infantil La Paz, Madrid, España.

Martínez, M., García, M. (2012). La crianza como objeto de estudio actual desde el modelo transaccional. Revista Latinoamericana de Ciencias Sociales, Niñez y Juventud, 10 (1), pp. 169-178

MINSAL (2004) Normas Técnicas de Estimulación y Evaluación del Desarrollo Psicomotor del Niño y la Niña menor de 6 años. Programa de Salud del Niño. Santiago, Chile: Gobierno de Chile

MINSAL (2006) Encuesta de Calidad de Vida y Salud Chile 2006. Subsecretaría de Salud Pública. Santiago, Chile: Gobierno de Chile. Recuperado de: http://epi.minsal.cl/wp-content/uploads/2012/07/ Informe-Final-Encuesta-de-Calidad-de-Vida-y-Salud-2006.pdf

MINSAL (2008) Manual para el Apoyo y Seguimiento del Desarrollo Psicosocial de los niños y niñas de 0 a 6 años. Santiago, Chile: Gobierno de Chile.

MINSAL (2010). Los Objetivos Sanitarios para la Década 2000-2010. Santiago, Chile: Gobierno de Chile.

MINSAL (2011) Estrategia Nacional de Salud Para el cumplimiento de los Objetivos Sanitarios de la Década 2011-2020. Santiago, Chile: Gobierno de Chile.

MINSAL (2011) Orientaciones Técnicas para la atención psicosocial de los niños y niñas hospitalizados en servicios de neonatología y pediatra. Plan Nacional de Salud de la Infancia, Programa de Apoyo al Desarrollo Biopsicosocial Santiago, Chile: Gobierno de Chile.

MINSAL (2011) Secretaría ejecutiva Chile Crece Contigo. Departamento de Estadísticas e Información en Salud. Recuperado el 10/10/2014 en: https://spreadsheets2.google.com/spreadsheet/pub

MINSAL (2012). Afiche Epidemiológico Región Metropolitana. Recuperado de: http://www.chccsalud.cl/

MINSAL (2012). Orientaciones técnicas para las modalidades de apoyo al desarrollo infantil: Guía para los Equipos Locales. Subsitema de Protección Integral a la Infancia, Equipo Chile Crece Contigo. Santiago, Chile: Gobierno de Chile.

Puchol Esparza, D. (2011). El modelado: definición, factores clave y ámbitos de aplicación en psicoterapia. PSICOLOGÍA ONLINE.

\footnotetext{
http://www.psicologia-online.com/colaboradores/dpuchol/ modelado.shtml
}

${ }^{R}$ Odriguez, S., Arancibia, V., Undurraga, C. (1989) EEDP. Escala de Evaluación de Desarrollo Psicomotor de 0 a 24 meses. Editorial Galdoc, Chile.

${ }^{R}$ Odriguez, S., Arancibia, V., Undurraga, C. (Ed) (2012) EEDP Escala de Evaluación del Desarrollo Psicomotor de 0 - 24 meses. Santiago,Chile: Cronopios.

Siegel, R., Naishadham, D., \& Jemal, A. (2013). Cancer statistics, 2013. CA: a cancer journal for clinicians, 63(1), 11-30.

Thompson, R. A. (2009). The handbook of child life: A guide for pediatric psychosocial care. Charles $C$ Thomas Publisher.

vargas, P. (2000). Cáncer en pediatría: Aspectos generales. Revista chilena de pediatría, 71(4), 283-295. 